\title{
CT-1 induces angiogenesis by regulating the ADMA/DDAH Pathway
}

\author{
Zhen Zhong Zheng ${ }^{\mathrm{a}}$, Xiao Tian Fu ${ }^{\mathrm{a}, \mathrm{b}}$, Jin Liang ${ }^{\mathrm{a}}$, Zhi Bing Guo ${ }^{\mathrm{a}}$
}

\begin{abstract}
Background. Cardiotrophin-1 (CT-1), a member of the IL-6 superfamily, is elevated in the serum of patients with ischemic and valvular heart disease. In this study, we hypothesized that CT-1 induces endothelial cell angiogenesis and that the ADMA/DDAH pathway plays an important role in the process.

Methods. pEGFP-N1-CTF1-GFP and pEGFP-N1 were constructed and used to transiently transfect to HUVECs, mediated by LipofectamineTM 2000. After transfection, the expression of CT-1 was examined by qRT-PCR and western blotting. Endothelial cell proliferation assay was evaluated using the 3-[4,5-dimethylthiazol-2-yl]-2,5 diphenyltetrazolium bromide (MTT) method. Migration assay was performed using transwell, tube formation test was examined on Matrigel, eNOSmRNA expression was assayed by qRT-PCR, DDAH I, DDAHII and VEGF expression were detected by western blotting, the level of ADMA and the activity of DDAH were measured by High Performance Liquid Chromatography, NOS activity and the concentration of $\mathrm{NO}$ were assayed by $\mathrm{L}-[3 \mathrm{H}]$ citrulline production from $\mathrm{L}-[3 \mathrm{H}]$ arginine.

Results. Overexpression of CT-1, increased endothelial cell proliferation, migration and formation of blood vessels, upregulated the expression of eNOSmRNA, DDAHI, DDAHII and VEGF, elevated the activity of DDAH and NOS, decreased the level of ADMA and promoted NO synthesis. In contrast, ADMA partially inhibited the effects of CT-1 induction.

Conclusions. Overexpression of CT-1 increases cell proliferation, migration and formation of blood vessels. This result also suggests that CT-1 may regulate angiogenesis through the ADMA/DDAH pathway.
\end{abstract}

Key words: cardiotrophin-1 (CT-1), ADMA, DDAH, eNOS, angiogenesis

Received: November 11, 2014; Accepted: February 18, 2015; Available online: March 1, 2015 http://dx.doi.org/10.5507/bp.2015.009

${ }^{a}$ Department of Cardiology, The First Afficiated Hospital of Nanchang University, Nanchang, Jiangxi, 330006, China ${ }^{b}$ Department of Electrocardiography, Hubei Provincial Maternal and Child Health Institute, Hubei, Wuhan, 430000, China Corresponding author: Zhen Zhon Zheng, e-mail: greateful@163.com

\section{INTRODUCTION}

Cardiotrophin-1 (CT-1) is a member of the IL-6 superfamily and is elevated in the serum of patients with ischemic heart disease and valvular heart disease. It also induces cardiomyocyte hypertrophy in vitro ${ }^{1}$. Past studies showed that short-term administration of CT-1 could improve the function of cirrhotic liver remnants and stimulate liver regeneration through promotion of angiogenesis and cell proliferation ${ }^{2}$. However, to date, we have found no study that showed CT-1 is involved in endothelial cell angiogenesis. For this reason, we hypothesized that cardiotrophin-1 may be involved in angiogenesis and may be a potential target for treating ischemic heart disease.

Angiogenesis is the formation of new capillaries from existing blood vessels. It is a multistep process that involves dissociation of endothelial cells (EC) from adjacent pericytes, remodelling of the extracellular matrix, proliferation and migration of EC and capillary differentiation. Whether CT-1 stimulates endothelial cell angiogenesis remains an open question. The ADMA/DDAH pathway influences angiogenesis by affecting expression of vascular endothelial growth factor (VEGF). In one study ADMA/DDAH was shown to influence VEGF responses by affecting NO bioavailability ${ }^{2}$. NO signaling is critically involved in VEGF-mediated chemotaxis and angiogenesis. Whether the ADMA/DDAH pathway regulates CT-1-Induced angiogensis is still unknown
The purpose of this study was hence to s explore whether CT-1 could induce angiogenesis through regulation of the ADMA/DDAH pathway.

\section{MATERIALS AND METHODS}

pEGFP-N1-CTF1-GFP and pEGFP-N1 were provided by Jikai company (Shanghai, China), Dulbecco's modified Eagle's medium (DMEM) culture medium and fetal bovine/calf serum were purchased from Hyclone company (USA). Lipofectamine 2000 and TRIzol Reagent were from Invitrogen Co (USA). ADMA and EDTA were purchased from Sigma Company (USA). Transwell Permeable Supports were from CORNING company. Matrigel Basement Membrane Matrix was from BD Biosciences company. Mouse anti-human CT-1 was from abcam company (USA). Mouse anti-human VEGF was from Santa Cruz Biotechnology (USA). Enhanced chemiluminescent (ECL) substrate was from Pierce (USA). Rabbit anti-human $\beta$-actin antibody was purchased from Zhongsha Biotechnology (China). Goat anti-human DDAHI and DDAHII antibody were from Santa Cruz Biotechnology (USA). eNOS and NO detecting Kits were from Jiancheng company (China). 


\section{Cell culture and plasmid transfection}

The human umbilical vein cells were provided by Nanchang University and cultured in DMEM supplemented with $10 \%$ fetal serum bovine (FBS), penicillin and streptomycin, human umbilical vein cells were grown on 6 -well plates (70-90\% confluent) and pre-incubated in OptiMem media for $15 \mathrm{~min}$ at $37{ }^{\circ} \mathrm{C}$. We divided this experiment into four groups; (1) HUVECs group: normal control; (2) GFP group: transfected with pEGFP-N1; (3) CT-1 group: transfected with pEGFP-N1-CTF1-GFP; (4) CT-1 +ADMA group: after transfecting with pEGFP-N1CTF1-GFP for $24 \mathrm{~h}$, HUVECs were cultured with 100 $\mu$ mol/LADMA. The plasmids $(1 \mu \mathrm{g})$ was mixed with Lipofectamine 2000 ( $5 \mu \mathrm{L} /$ well) and incubated for $15 \mathrm{~min}$ at $37{ }^{\circ} \mathrm{C}$. The lipid-coated DNA was then added to each well containing $2 \mathrm{~mL}$ of DMEM media and incubated for $6 \mathrm{~h}$. At the end of this period, the media were removed and replaced with complete media, after which the human umbilical vein cells were lysed or used in migration assays.

\section{Proliferation assay}

Proliferation assay performed as previously described $^{4,5}$. In brief, the cells were transfected with plasmids mediated by Lipofectamine 2000 in 6-well plates in triplicate, and $24 \mathrm{~h}$ later the cells were trypsinized and seeded in 96-well plates in triplicate $\left(1 \times 10^{4}\right.$ cells/well $)$. After attachment, the cells were exposed to DMEM with $0.5 \% \mathrm{FBS}$ for $48 \mathrm{~h}$, and then cell proliferation was evaluated using the 3-[4,5-dimethylthiazol-2-yl]-2,5, diphenyltetrazolium bromide (MTT) (Sigma, USA) colorimetric assay. Absorbance was measured at $570 \mathrm{~nm}$ for each well using a microplate reader (Bio-Tek Instruments, USA) according to the manufacturer's protocol.

\section{Migration assay}

Cell migration assay was performed as previously described $^{4,5}$. It was done using Boyden transwell chambers (6.5 $\mathrm{mm}$ in diameter). Briefly, $200 \mu \mathrm{L}$ DMEM containing $10 \%$ FBS was added to the bottom well. Cells were resuspended in the appropriate buffer at a concentration of $1 \times 10^{5}$ cells $/ \mathrm{mL}$, and $600 \mu \mathrm{L}$ cell suspension was added to the top well of the Transwell chambers. Between the bottom well and the top well, there was a filter. After incubation at $37^{\circ} \mathrm{C}$ in $5 \% \mathrm{CO} 2$ conditions, the cells that had not migrated were removed from the upper surface of the filters using cotton swabs, and those that migrated to the lower surface of the filters were fixed in methanol and stained with hematoxylin. Migration was determined by counting the cell number with a microscope at $\times 400$. Five visual fields were chosen randomly for each assay. The average number of the migrating cells in the 5 fields was taken as the cell migration number of the group. The assays were repeated 3 times.

\section{Tube formation analysis}

Tube formation analysis performed as previously described $^{4,5}$. In vitro formation of capillary like tube structures was examined on matrigel. Matrigel $(0.5 \mathrm{~mL})$ was polymerized on 24 -well plates and $5 \times 10^{4}$ cells were then plated in full-growth media for $1 \mathrm{~h}$. Once the cells were seeded, the media was replaced with media containing $0.5 \%$ serum. Tube formation was visualized using an inverted microscope (Olympus, Japan) equipped with digital imaging. For each treatment, 10 high power field images were captured and the area of endothelial tubes and networks formed was quantified using the Scion Image Analysis System (Frederick, Maryland, USA) with background subtraction.

\section{Measurement of eNOS activity and NO level}

According to the protol and previously described ${ }^{4,5}$, the activity of eNOS was assayed by L-[3H] citrulline production from $\mathrm{L}-[3 \mathrm{H}]$ arginine.In brief, cell lysates $(100 \mu \mathrm{L})$ were incubated in $50 \mathrm{mmol} / \mathrm{L}$ Tris- $\mathrm{HCl}$ buffer ( $\mathrm{pH} 7.5$ ) containing the cofactors $100 \mathrm{nmol} / \mathrm{L}$ calmodulin, $2.5 \mathrm{mmol} / \mathrm{L} \mathrm{CaCl} 2,1 \mathrm{mmol} / \mathrm{L}$ nicotinamide adenine dinucleotide phosphate (NADPH), $10 \mu \mathrm{mol} / \mathrm{L}$ tetrahydrobiopterin, $1 \mathrm{mmol} / \mathrm{L}$ dithiotreitol, and the substrate, $1 \mu \mathrm{Ci}$ $\mathrm{L}-[3 \mathrm{H}]$ arginine, for $15 \mathrm{~min}$ at $37^{\circ} \mathrm{C}$. After the incubation period, the reaction was quenched by the addition of $1 \mathrm{~mL}$ of stop buffer [20 mmol/L N-2-hydroxyethylpiperazineN'-2-ethane sulfonic acid (HEPES), $2 \mathrm{mmol} / \mathrm{L}$ EDTA, and $0.2 \mathrm{mmol} / \mathrm{L}$ ethyleneglycol-bis-( $\beta$-aminoethyl ether)$\mathrm{N}, \mathrm{N}, \mathrm{N}^{\prime}, \mathrm{N}^{\prime}$-tetraacetic acid (EGTA), pH 3]. The reaction mixture was applied to a $1 \mathrm{~mL}$ column containing Dowex AG 50WX-8 resin (Na+ form, Bio-Rad, Hercules, CA, USA) that had been pre-equilibrated with the stop buffer. $\mathrm{L}-[3 \mathrm{H}]$ citrulline was eluted twice with $0.5 \mathrm{~mL}$ of stop buffer and radioactivity was determined by liquid scintillation counting.

Nitrite/nitrate levels, an indicator of NO synthesis, were measured by Griess reaction. After conversion of nitrate to nitrite in the presence of nitrate reductase and cofactors, $100 \mu \mathrm{L}$ of medium were mixed with $100 \mu \mathrm{L}$ griess reagent ( $1 \%$ sulfanilamide; $1 \%$ naphthylethylenediamine in $2 \%$ phosphoric acid) and optical density was determined at $540 \mathrm{~nm}$.

\section{Western blot analysis}

Cells were scraped off the plates and lysed in RIPA buffer (50 mM Tris-HCl, pH 8.0, $150 \mathrm{mM} \mathrm{NaCl,} \mathrm{1 \%}$ Nonidet P-40, 0.5\% deoxycholic acid, $0.1 \%$ sodiumdodecyl sulfate). Cell lysates (50 g protein) were electrophoresed on SDS-PAGE gel and transferred to polyvinyldifluoride membrane. Membranes were incubated with antibodies against CT-1, VEGF, DDAHI and DDAHII. After incubation, the corresponding secondary antibody signals were detected by the enhanced chemiluminescence reagents. The intensities of the various protein bands were quantified by densitometry.

\section{ADMA level and DDAH activity}

To determine the level of DDAH activity, ADMA concentration was quantified using high-performance liquid chromatography (HPLC) following the exogenous addition of ADMA to cells. After infection, the cells were rinsed with PBS and incubated with ADMA (100 $\mu \mathrm{mol} / \mathrm{L})$ for $2 \mathrm{~h}$, and the residual ADMA was then removed by PBS 
washes. The cells were scraped in PBS, sonicated on ice, and centrifuged to pellet cellular debris. Crude lysates were treated with NG-monomethyl-1-arginine (1-NMMA; Sigma) as an internal standard and subjected to solidphase extraction and HPLC methods. Data are reported as the peak area ratio of ADMA to 1-NMMA, normalized to the protein concentration of each sample. The protein concentration of the crude lysates was determined using the bicinchoninic acid method (Pierce, Rockford, IL).

\section{Quantitative real-time PCR}

According to previously described ${ }^{6}$. After transduction and/or stimulation, the cells were lysed and the RNA was isolated according to TRIzol Reagent protocol. cDNA was synthesized from $1 \mu \mathrm{g}$ of total RNA using random hexamers and MultiScribe Reverse Transcriptase (Applied Biosystems, Foster City, CA). Quantitative real-time PCR was carried out using a HT7000 cycler (Applied Biosystems). Primer/probe sets for CT1, endothelial NOS (eNOS). Real-Time PCR System (Stratagene,USA), using primers CT-1(ID: Hs-QRP2061); $\beta$-actin-Forward:5'- AGCGAGCAT CCCCCAAA GTT-3', $\beta$-actin-Reverse:5'-GGGCACGAAGGCTCATT-3'. eNOS-Forward:5'-ACG AGGAGTGGAAGTGGTTC3",eNOS-Reverse:5'-GAGATGGTCAAGTTGGG AGC3'. The reactions were performed at $95{ }^{\circ} \mathrm{C} 10 \mathrm{~min}, 40$ cycles of $95^{\circ} \mathrm{C} 15 \mathrm{~s}, 60{ }^{\circ} \mathrm{C} 20 \mathrm{~s}, 72{ }^{\circ} \mathrm{C} 1 \mathrm{~min}$, followed by melting curve analysis according to instrument documentation.All reactions were done in triplicates and the results were normalized by $\beta$-action.

\section{Statistical Analysis}

Data are expressed as means \pm SEM. Differences among groups were assessed by SPSS14. A probability value $<0.05$ was taken as statistically significant.

\section{RESULTS}

\section{Expression of CT-1 protein after pEGFP-N1-CTF1-GFP transfection}

pEGFP-N1-CTF1-GFP were identified by agarose gel electrophoresis, and electrophoresis generating a $268 \mathrm{bp}$ bp fragment, which was compared with the desired result (Fig. 1A). $\beta$-actin was used as an internal control to monitor equal protein sample loading (Fig. 1D,E), the results of $2^{-\Delta \Delta \mathrm{Ct}}$ showed that the expression of CT-1mRNA significantly increased in CT-1 group (Fig. 1B,C,E,F) and was more than $40.027 \pm 1.0977$ of HUVEC group, the difference is significant $(P<0.05)$. The expression of GFP group is $1.192 \pm 1.1149$ of HUVEC group but the difference is not significant $(P>0.05)$ (Fig. 1F). The results indicated thatpEGFP-N1-CTF1-GFP infection increased the expression of CT-1 mRNA.

\section{Expression of CT-1 protein after pEGFP-N1-CTF1-GFP transfection}

To observe the time course of the expression of CT-1 after transfection, we found that CD151 levels increased at 24,48 , or $72 \mathrm{~h}$, but significantly increased at $48 \mathrm{~h}(P<$ 0.05 ) (Fig 2B), so we select the $48 \mathrm{~h}$ time point to further study. We also observed that the expression of CT-1 in the
A
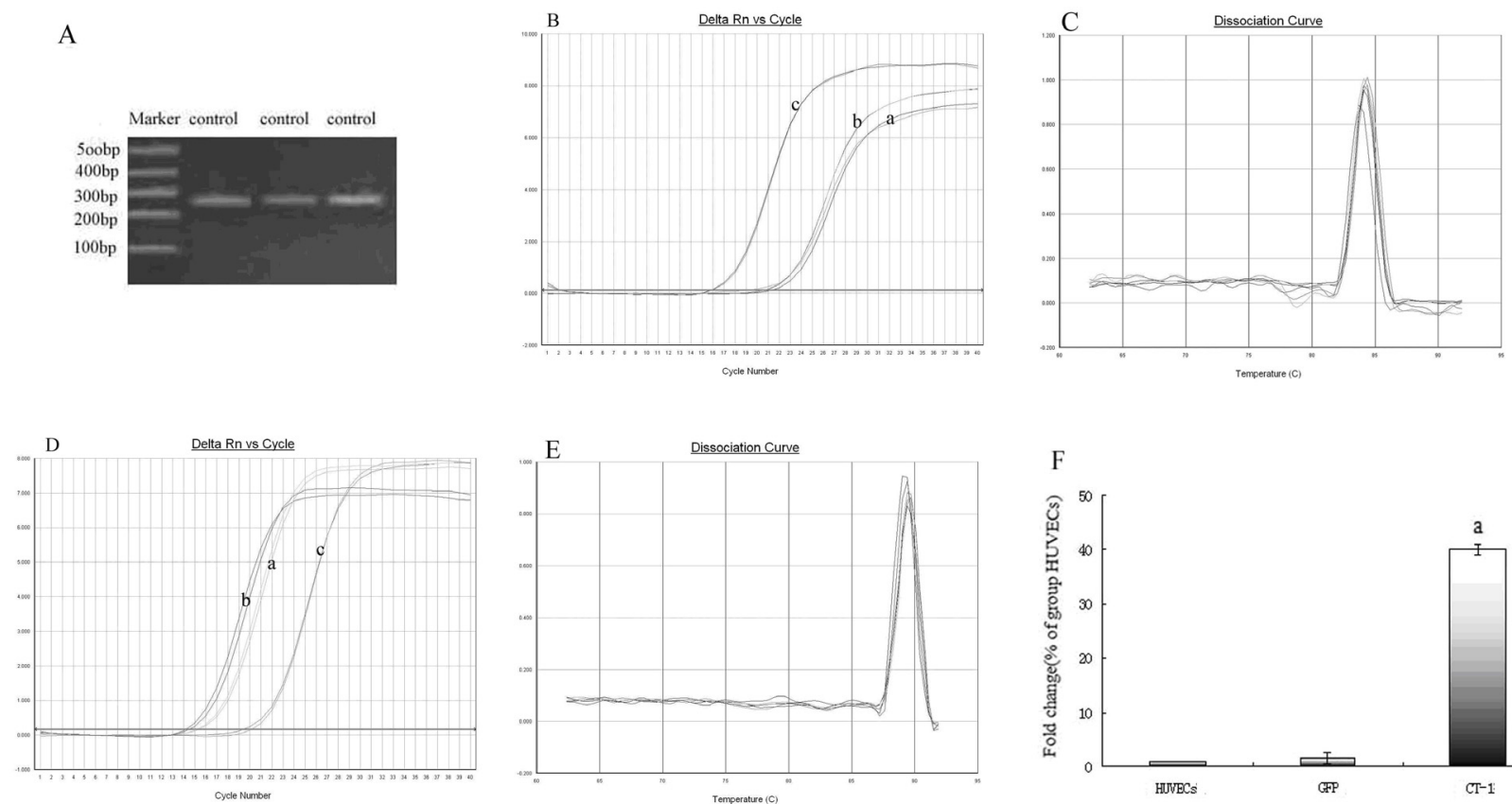

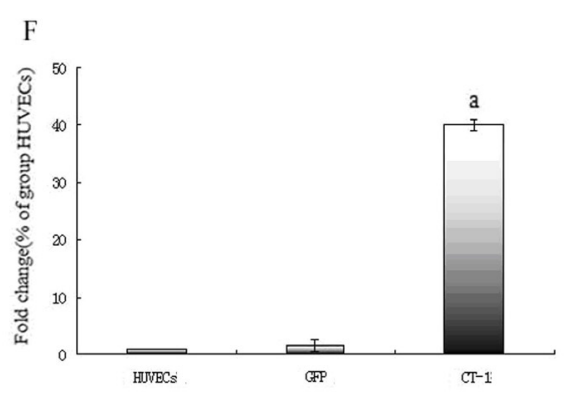

Fig. 1. The expression of CT-1 mRNA after pEGFP-N1-CTF1-GFP transfection. (A) CT-1 PCR product by agarose gel electrophoresis. (B) CT-1 Gene amplification curve. (C) CT-1 gene dissolution curve. (D) $\beta$-actin Gene amplification curve. (E) $\beta$-actin gene dissolution curve. (F) The results of $2^{-\triangle \Delta \mathrm{Ct}} \cdot \mathrm{a}-\mathrm{HUVEC}$ group, b-GFP group c-CT-1 group ${ }^{\mathrm{a}} P<0.05$ vs HUVECs group. 


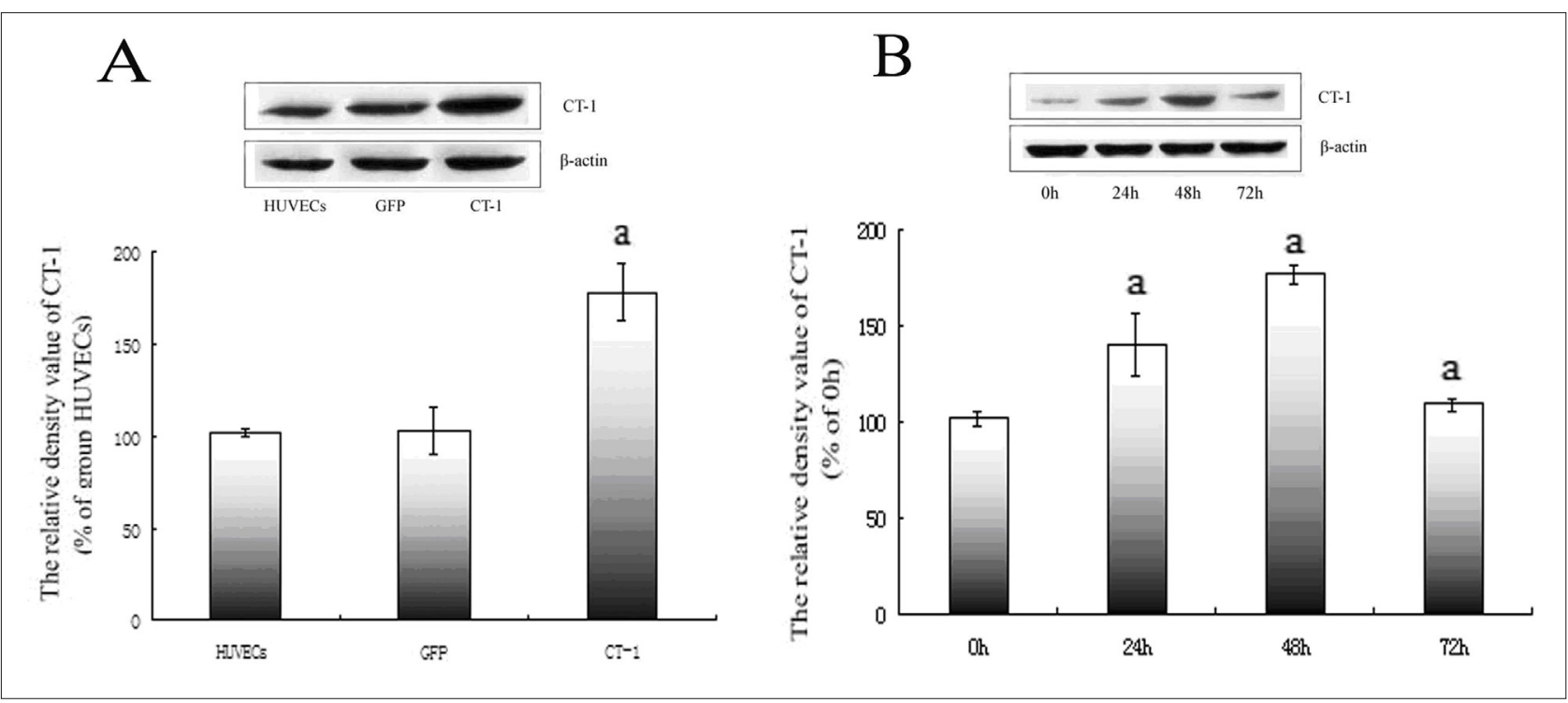

Fig. 2. Western blot analysis of the expression of CT-1. $\beta$-actin was used as an internal control to monitor equal protein sample loading. (A) Expression of CT-1 after transfection at $48 \mathrm{~h}$. (B) Expression of CT-1 after transfection at different times. ${ }^{\text {a }} P<0.05$ vs 0 h. $\mathrm{n}=3$. Mean \pm SD. ${ }^{\text {a }} P<0.05$ vs control and GFP groups.

cells significantly increased $(P<0.05)$ compared with the control and GFP groups $48 \mathrm{~h}$ after transfection (Fig. 2A).

\section{Proliferation assay at $48 \mathrm{~h}$ after transfection}

The difference in OD value is not significant $(P>$ $0.05)$ between HUVEC group $(0.2662 \pm 0.0044)$ and GFP group (0.2698 \pm 0.0049$)$. Compared with HUVEC groups, the OD value of CT-1 group $(0.4695 \pm 0.0039)$ significantly increased $(P<0.05)$.In addition, compared with HUVEC group, the OD value of CT-1+ADMA group $(0.3276 \pm 0.0072)$ significantly increased $(P<0.05)$ and the difference is significant $(P<0.05)$ between CT-1+ADMA group and CT-1 group, the proliferation of CT-1 group is more than CT-1+ADMA group. The results indicated that CT-1 promotes cell proliferation and ADMA partly inhibits cell proliferation.

\section{Migration assay at $48 \mathrm{~h}$ after transfection}

In transwell boyden chamber assay, the numbers of migrated cells are no significant between in GFP group (30.4 \pm 1.1402$)$ and HUVEC group $(31 \pm 1.8708)(P>0.05)$. The numbers of migrated cells of CT-1 group $(67 \pm 1.5811)$ increased significantly compared with HUVEC group $(P<$ 0.05 ) and the numbers of migrated cells of CT-1+ADMA group significant decreased compared with CT-1 group $(P<0.05)$. Results showed that CT-1 stimulated HUVEC migration, and ADMA attenuated CT-1 induced HUVEC migration (Fig. 2). These data suggest that CT-1 and AMDA play important roles in migration of HUVEC.

\section{Tube formation analysis}

Matrigel tests demonstrated that the vessel numbers of CT-1 group (9.75 \pm 0.9574$)$ increased significantly compared with HUVEC group $(4.5 \pm 0.5773)(P<0.05)$. The vessel numbers of CT-1+ADMA group also increased compared with HUVEC group $(7.5 \pm 0.9574)(P<0.05)$. The vessel numbers of GFP group is $(4.25 \pm 0.5)$ and

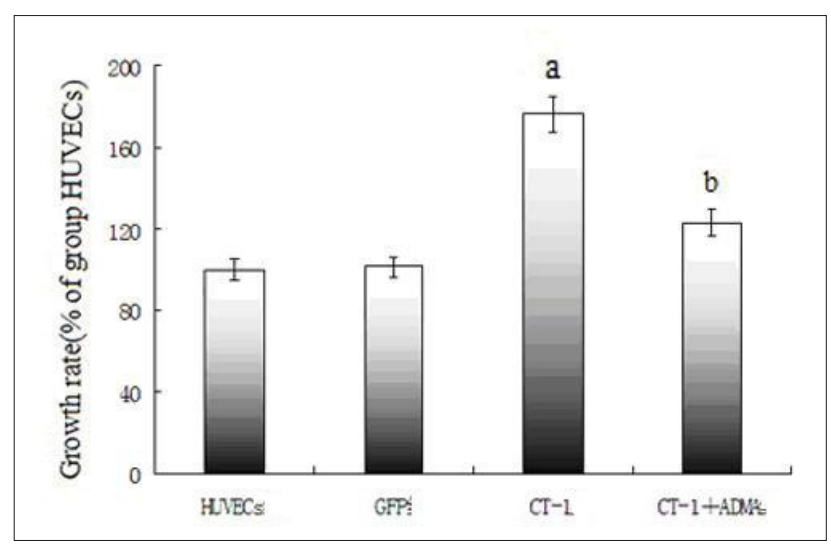

Fig. 3. Proliferation assay results at $48 \mathrm{~h}$ after transfection. ${ }^{\mathrm{a}} P<0.05$ vs HUVECs group, ${ }^{\mathrm{b}} P<0.05$ vs $\mathrm{CT}-1$ group.

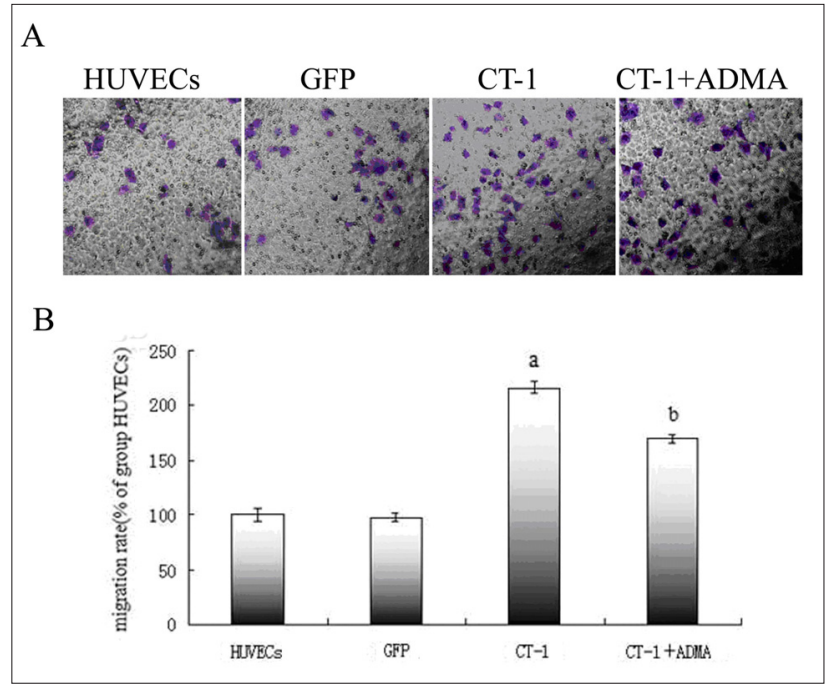

Fig. 4. Migration assay results at $48 \mathrm{~h}$ after transfection. (A) Representative photomicrographs at $48 \mathrm{~h}$ after transfection. (B) The migrated cell number in different group. ${ }^{\mathrm{a}} P<0.05$ vs HUVECs group, ${ }^{\mathrm{b}} P<0.05$ vs $\mathrm{CT}-1$ group. 
have no significance compared with HUVEC group $(P>$ $0.05)$. In addition, the vessel numbers of CT-1+ADMA group dramatically $(P<0.05)$ decreased compared with CT-1 group (Fig. 5). These data suggested that CT-1 enhanced capillary tube formation, which was inhibited by ADMA, indicating that ADMA played a critical role in CT-1-induced tube formation.

\section{Expression of eNOS mRNA after transfection}

The results of $2^{-\Delta \Delta \mathrm{Ct}}$ showed that the expression of eNOS mRNA in CT-1 group is $29.103 \pm 0.7543$ of HUVEC group, there is significance between the two groups $(P<$ $0.05)$. The expression of eNOS mRNA in CT-1+ADMA group is $8.6418 \pm 0.8363$ of HUVEC group, there is significance between the two groups $(P<0.05)$ and there is no significance between the HUVEC group and GFP group $(P>0.05)$ (Fig. 6). These data indicated that CT-1 gene transfer enhances eNOS mRNA expression, and ADMA inhibits CT-1-induced eNOS mRNA expression.

\section{The expression of DDAH I, DDAHII and VEGF after transfection}

Western blot analysis revealed that CT-1 gene transfection increased the expression of DDAHI, DDAHII and VEGF, whereas ADMA decreased the expression of DDAHI, DDAHII and VEGF (Fig. 7). Based on data above, we showed that DDAHI, DDAHII and VEGF are involved in the process. These data suggested CT-1 induced angiogenesis and DDAHI, DDAHII and VEGF may play roles in the process.

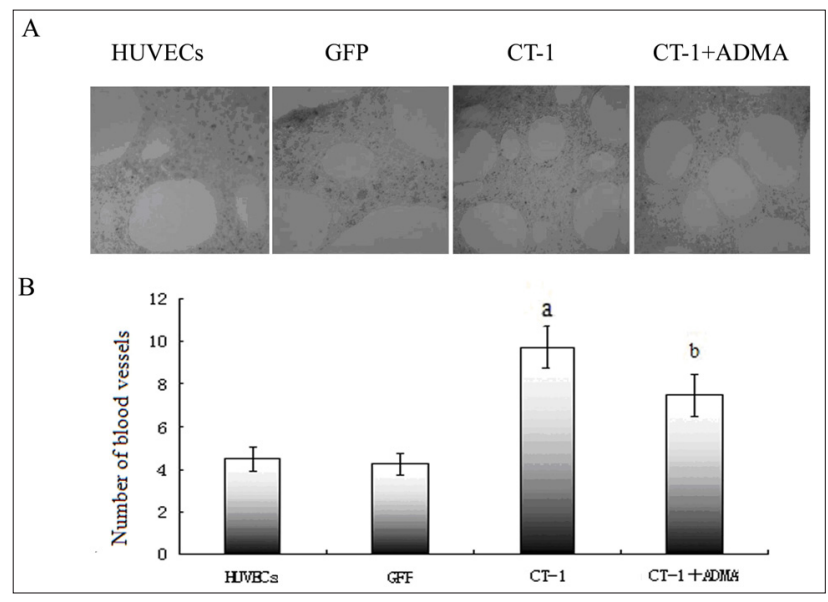

Fig. 5. Tube formation analysis. (A) Representative photomicrographs showing effects of CT-1 gene delivery on tube formation. (B) Quantitative analysis of the numbers of blood vessels in different group.

$P<0.05$ vs HUVECs group, ${ }^{\mathrm{b}} P<0.05$ vs $\mathrm{CT}-1$ group.

\section{ADMA level and DDAH activity at $48 \mathrm{~h}$ after transfection}

The level of ADMA was measured by High Performance Liquid Chromatography, results showed that ADMA level is $0.7429 \pm 0.0015 \mu \mathrm{mol} / \mathrm{L}$ in GFP group, and there was no significance between GFP group and HUVEC group $(0.7439 \pm 0.0045 \mu \mathrm{mol} / \mathrm{L})$. The level of ADMA in CT-1 group $(0.373 \pm 0.0016 \mu \mathrm{mol} / \mathrm{L})$ decreased significantly $(P<0.05)$. The level of ADMA in CT$1+$ ADMA group $(0.5767 \pm 0.0037 \mu \mathrm{mol} / \mathrm{L})$, and there was

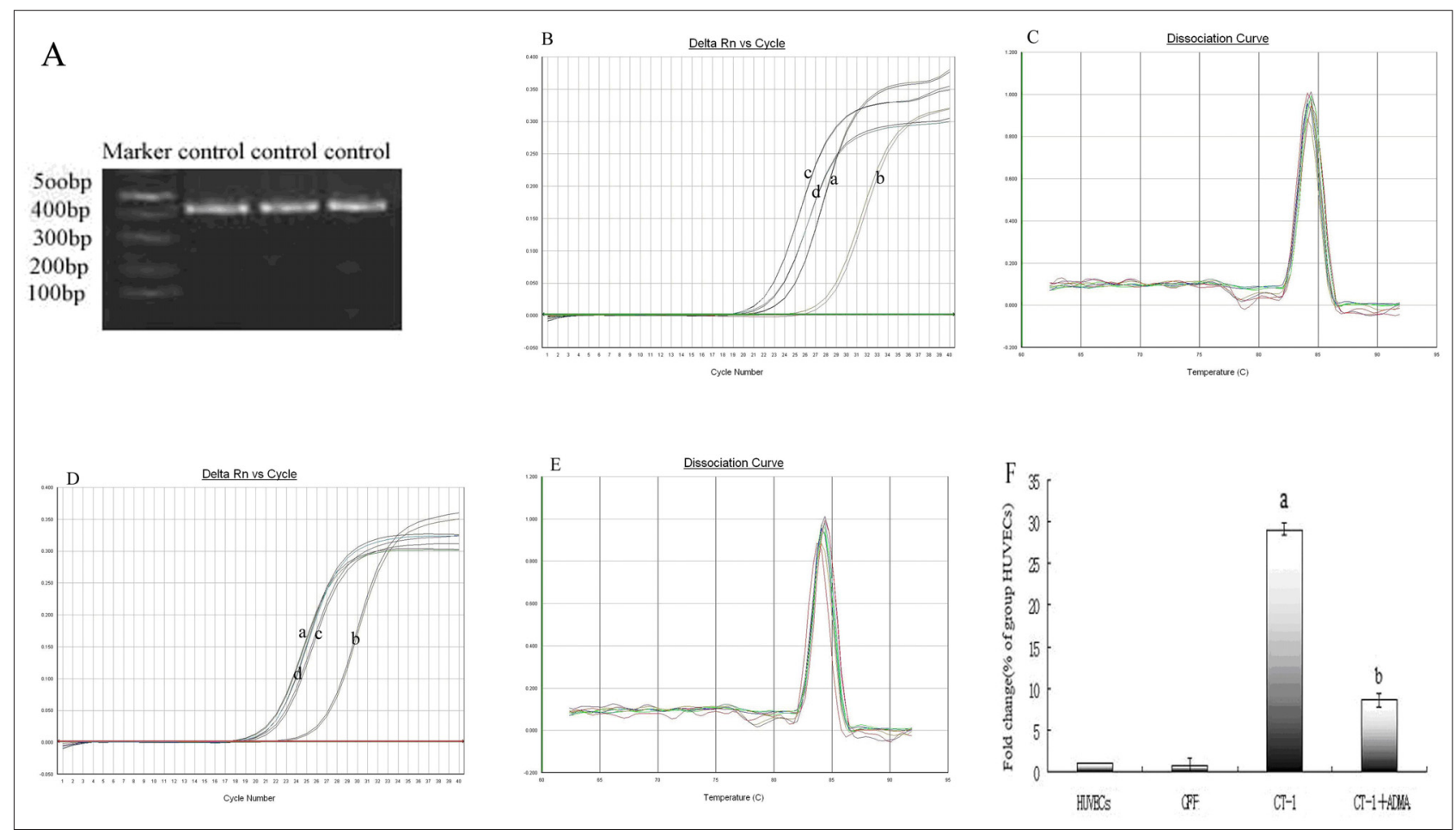

Fig. 6. The expression of eNOS mRNA after pEGFP-N1-CTF1-GFP transfection. (A) eNOS mRNA PCR product by agarose gel electrophoresis. (B) eNOS mRNA Gene amplification curve. (C) eNOS mRNA gene dissolution curve. (D) $\beta$-actin Gene amplification curve. (E) $\beta$-actin gene dissolution curve. (F) The results of $2^{-\triangle \triangle C t} \cdot a-H U V E C$ group. b-GFP group. c-CT-1 group. ${ }^{\text {a }} P<0.05$ vs HUVECs group. 


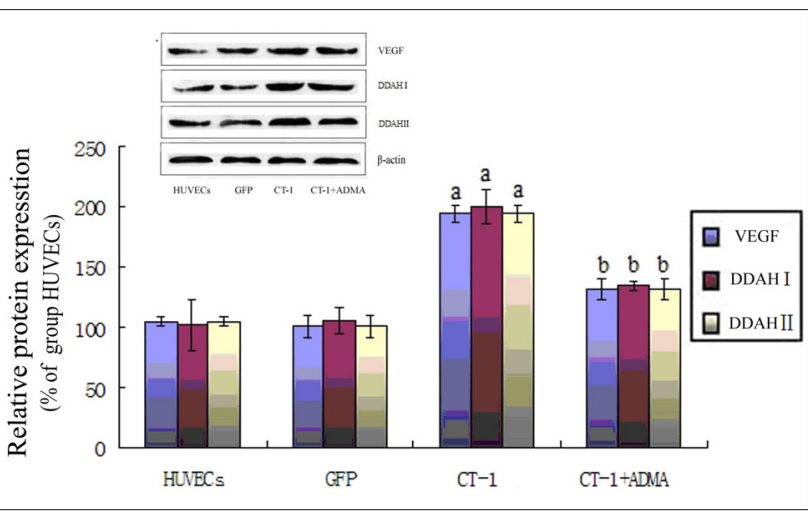

Fig. 7. Western blot analysis of the expression of DDAHI, DDAHII and VEGF.

$\beta$-actin was used as an internal control to monitor equal protein sample loading. (A) Expression of DDAHI, DDAHII and VEGF after transfection at $48 \mathrm{~h}$. (B) Expression of DDAHI, DDAHII and VEGF after transfection in different groups. ${ }^{a} P<0.05$ vs HUVEC group. $\mathrm{n}=3$. Mean $\pm \mathrm{SD} \cdot{ }^{\mathrm{b}} P<0.05$ vs $\mathrm{CT}-1$ groups.

significant compared with CT-1 group $(P<0.05)$ (Fig. $8 \mathrm{~A})$.These data indicated that overexpression of CT-1 decreased AMDA level.

Results showed that DDAH activity significantly increased in CT-1 group compared with HUVEC group ( $P$ $<0.05)$, and DDAH activity significantly increased in CT$1+$ ADMA group compared with HUVEC group $(P<0.05)$. There was no significance between the activity of DDAH in GFP group and HUVEC group $(P>0.05)$ (Fig. 8B).These results indicated CT-1 increase the activity of DDAH, and exogenous ADMA inhibited the activity of DDAH.

\section{NOS activity and NO level at $\mathbf{4 8 h}$ after transfection}

Results showed that NOS activity of CT-1 group increased compared HUVEC group $(P<0.05)$, and NOS activity of CT-1+ADMA group also increased compared HUVEC group $(P<0.05)$. There was no significance between the activity of NOS activity in GFP group and HUVEC group $(P>0.05)$ (Fig. 9A). These results indicated CT-1 increase the activity of NOS, and exogenous ADMA inhibited the activity of NOS.

In addition, results showed that NO level is $16.4432 \pm 0.2873 \mu \mathrm{mol} / \mathrm{L}$ in CT-1 group, and there was significant $(P<0.05)$ compared with HUVEC group $(5.5262 \pm 0.0758 \mu \mathrm{mol} / \mathrm{L})$. NO level of CT-1+ADMA group is $9.8169 \pm 0.9227 \mu \mathrm{mol} / \mathrm{L}$, and significantly increased compared with HUVEC group $(P<0.05)$. There were no significant between GFP group (5.5666 \pm 0.1526 $\mu \mathrm{mol} / \mathrm{L})$ and HUVEC group $(P>0.05)$ (Fig. 9B). These data indicated that overexpression of CT-1 decreased AMDA level.

\section{DISSCUSION}

Cardiotrophin-1 (CT-1) is a member of the interleukin-6 super family of cytokines. A large number of experimental studies demonstrate that CT-1 may induce

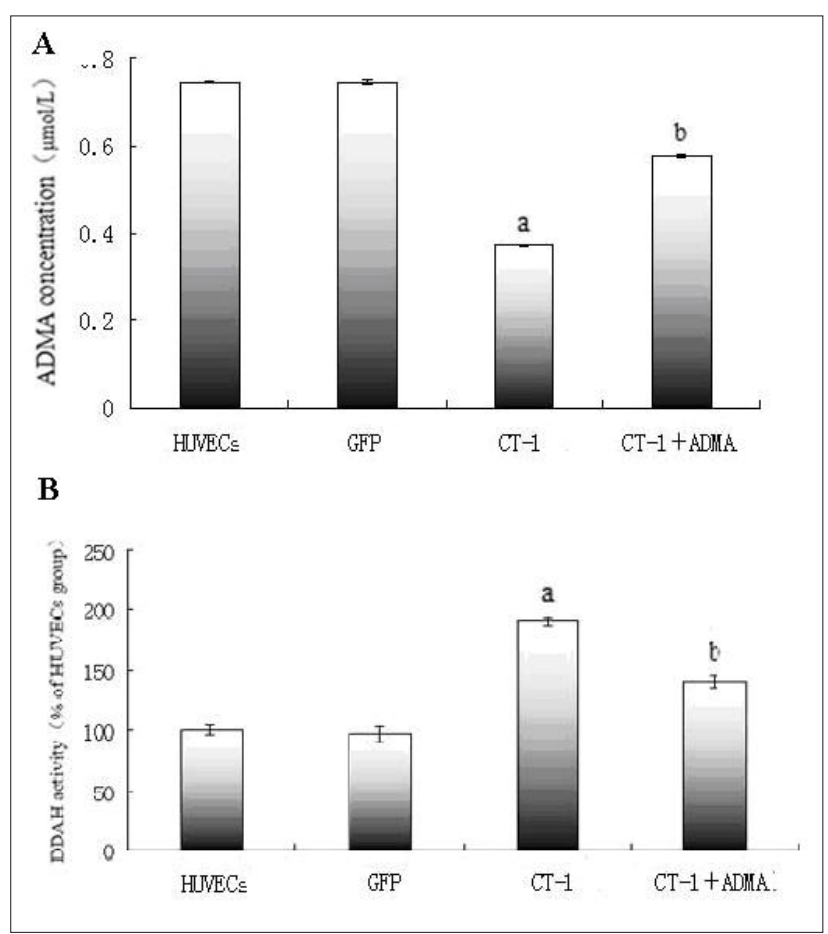

Fig. 8. ADMA level and DDAH activity at $48 \mathrm{~h}$ after transfection.

(A) The AMDA level at $48 \mathrm{~h}$ after transfection. (B) The activity of DDAH at $48 \mathrm{~h}$ after transfection. ${ }^{a} P<0.05$ vs HUVEC sgroup, ${ }^{\mathrm{b}} P<<0.05$ vs CT-1 group.

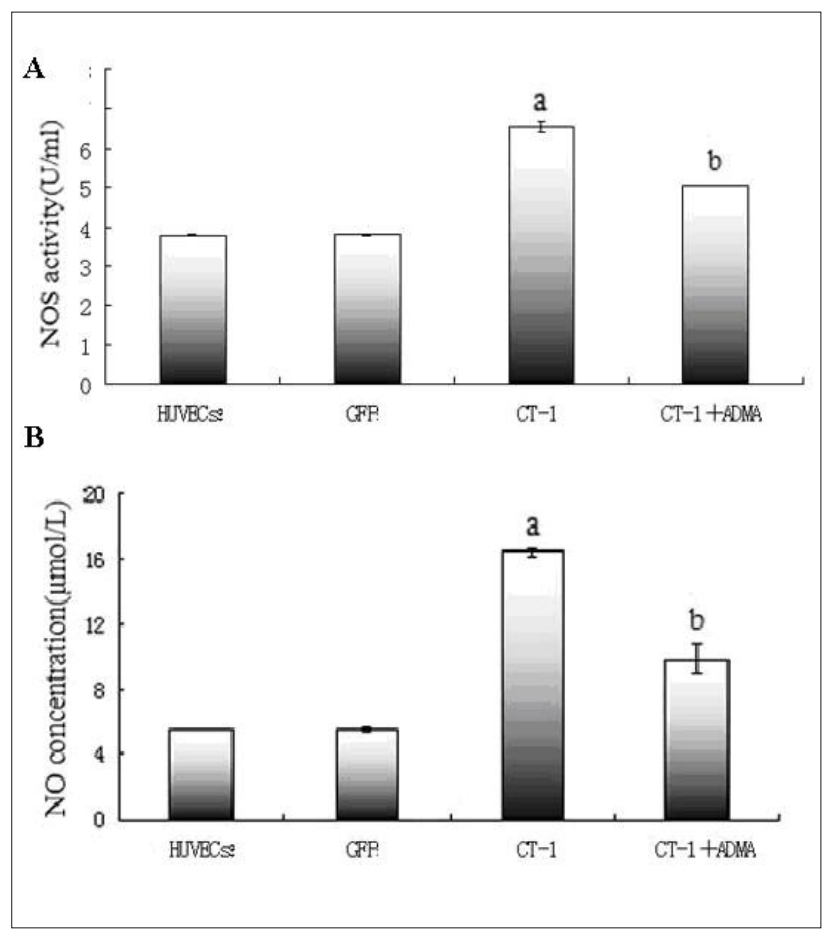

Fig. 9. NOS activity and NO level at $48 \mathrm{~h}$ after transfection. (A) The activity of NOS at $48 \mathrm{~h}$ after transfection. (B) The NO level at $48 \mathrm{~h}$ after transfection.

${ }^{\mathrm{a}} P<0.05$ vs HUVEC sgroup, ${ }^{\mathrm{b}} P<0.05$ vs $\mathrm{CT}-1$ group. 
myocardial hypertrophy ${ }^{1,7}$. It also has been shown that the expression of CT-1 in the post-MI heart issignificantly elevated ${ }^{8}$. CT-1 has been shown to mainly associate with myocardial hypertrophy ${ }^{9}$, but it was not clear whether CT1-mediated endothelial cells angiogenesis and the mechanism involved is not known.

A series of signal transduction pathways has been shown to be involved in the regulation of angiogenesis. The ADMA/DDAH pathway is one of these. Methylated analogues of arginine such as asymmetric dimethylarginine (ADMA) are competitive inhibitors of NO synthesis. Free ADMA is found in plasma and urine of healthy individuals and is synthesised by the post-translational methylation of protein arginine residues and liberated upon their hydrolysis. The intracellular concentration of ADMA reaches levels sufficient to inhibit NO synthesis and can be modulated by changes in the activity of the enzyme dimethylarginine dimethylaminohydrolase (DDAH). DDAHI predominates in tissues that express the neuronal isoform of NOS, while DDAHII distribution mainly follows endothelial NOS ( ref. $^{10}$ ). Studies have proved that nitric oxide (NO), produced from arginine by the nitric oxide synthases (NOS), is a crucial signalling molecule and regulator of angiogenesis. NO enhances vascular permeability, induces extracellular matrix degradation, endothelial cell proliferation and migration ${ }^{11}$ and stimulates the expression of vascular endothelial growth factor (VEGF) while also mediating many of its angiogenic effects ${ }^{12}$. Dysfunction of DDAH and elevated levels of its substrate ADMA have been implicated in pathological conditions including hypertension, pre-eclampsia, renal failure and atherosclerosis. High levels of ADMA have been correlated with impaired angiogenesis in hypercholesterolemic mice ${ }^{13}$. In conclusion, ADMA/ DDAH plays critical role in angiogensis.

\section{CONCLUSION}

In this study, an eukaryotic expression vector for CT-1 was constructed successfully and HUVECs were transfected with it. We have analyzed the function of CT-1 on endothelial cells proliferation, migration and formation of blood vessels. We hypothesized that CT-1 may activate ADMA/DDAH pathway and exert the function of angiogenesis. We demonstrate a novel mechanism by which a reduction in ADMA, increased DDAH activity, as a result of CT-1 overexpression. CT-1 stimulates endothelial cell proliferation and migration and angiogenesis through increased NO and VEGF expression. Increased ADMA level inhibits CT-1-induced endothelial cell proliferation and migration, at the same time decreases the expression of DDAH activity, NO and VEGF expression.

These data indicate the ADMA/DDAH pathway regulates CT-1-induced angiogensis. We showed for the first time that CT-1 induces angiogenesis and may be a target for treating ischemic heart disease.

\section{ABBREVIATION}

CT-1, Cardiotrophin-1; GFP, Green fluorescent protein; ADMA, Asymmetric Dimethylarginine; DDAH, Dimethylarginine Dimethylaminohydrolase; eNOS, endothelial nitric oxide synthase.

Acknowledgement: This work was supported by a grant from the Education Department Foundation of Jiangxi Province (NO. GJJ09423).

Author contributions: All authors contributed equally to preparing the manuscript.

Conflict of interest statement: None declared.

\section{REFERENCES}

1. Freed DH, Cunnington RH, Dangerfield AL, Sutton JS, Dixon IM. Emerging evidence for the role of cardiotrophin-1 in cardiac repair in the infarcted heart. Cardiovasc Res 2005;65(4):782-92.

2. Yang ZF, Lau CK, Ngai P, Lam SP, Ho DW, Poon RT, Fan ST. Cardiotrophin-1 enhances regeneration of cirrhotic liver remnant after hepatectomy through promotion of angiogenesis and cell proliferation. Liver Int 2008;28(5):622-31.

3. Fiedler LR, Bachetti T, Leiper J, Zachary I, Chen L, Renné T, WojciakStothard B. The ADMA/DDAH Pathway Regulates VEGF-Mediated Angiogenesis.Arterioscler Thromb Vasc Biol 2009;29:2117-24.

4. Zheng ZZ, Liu ZX. CD151 gene delivery increases eNOS activity and induces ECV304 migration, proliferation and tube formation. Acta Pharmacologica Sinica 2007;28(1):66-72.

5. Zheng ZZ, Liu ZX. Activation of the phosphatidylinositol 3-kinase/ protein kinase Akt pathway mediates CD151-induced endothelial cell proliferation and cell migration. Int J Biochem Cell Biol 2007;39(2):340-8.

6. Zheng ZZ, Wang L, Wu Y, Wang H, Gao D, Fa Y, Wei Y, Wang M, Zheng Z, Peng J. Fibrinogen-like protein 2 Gene silencing activates angiopoietin/Tie system and induces Myocardial Microvascular Endothelial Cells proliferation and cell migration. Biomedical Research 2012;23(1): 37-42.

7. López N, Díez J, Fortuño MA. Differential hypertrophic effects of cardiotrophin-1 on adult cardiomyocytes from normotensive and spontaneously hypertensive rats. J Mol Cell Cardiol 2006;41(5):90213.

8. Freed DH, Moon MC, Borowiec AM, Jones SC, Zahradka P, Dixon IM. Cardiotrophin-1: expression in experimental myocardial infarction and potential role in post-MI wound healing. Mol Cell Biochem 2003;254(1-2):247-56.

9. He-nan Z, Yan W, Miao-na J, Cai-hua Z, Shen L, Yu-jie J. Relation of Cardiotrophin-1 (CT-1) and cardiac transcription factor GATA4 expression in rat's cardiac myocytes hypertrophy and apoptosis. Pathol Res Pract 2009;205(9):615-25.

10. Leiper JM, Santa Maria J, Chubb A, MacAllister RJ, Charles IG, Whitley GS, Vallance P. Identification of two human dimethylarginine dimethylaminohydrolases with distinct tissue distributions and homology with microbial arginine deiminases. Biochem J 1999;343 Pt 1:209-14.

11. Jang JJ, Ho HK, Kwan HH, Fajardo LF, Cooke JP. Angiogenesis is impaired by hypercholesterolemia: role of asymmetric dimethylarginine. Circulation 2000;102(12):1414-9.

12. Fukumura D, Yuan F, Endo M, Jain RK. Role of nitric oxide in tumor microcirculation. Blood flow, vascular permeability, and leukocyteendothelial interactions. Am J Pathol. 1997;150(2):713-25.

13. Papapetropoulos A, Desai KM, Rudic RD, Mayer B, Zhang R, Ruiz-Torres MP, García-Cardeña G, Madri JA, Sessa WC. Nitric oxide synthase inhibitors attenuate transforming-growth-factorbeta 1-stimulated capillary organization in vitro. Am J Pathol 1997;150(5):1835-44. 